\title{
ASYMPTOTIC DECAY OF NONOSCILLATORY SOLUTIONS OF GENERAL NONLINEAR DIFFERENCE EQUATIONS
}

\author{
E. THANDAPANI, S. LOURDU MARIAN, and JOHN R. GRAEF
}

(Received 10 November 2000)

\begin{abstract}
The authors consider the $m$ th order nonlinear difference equations of the form $D_{m} y_{n}+q_{n} f\left(y_{\sigma(n)}\right)=e_{i}$, where $m \geq 1, n \in \mathbb{N}=\{0,1,2, \ldots\}, a_{n}^{i}>0$ for $i=1,2, \ldots, m-1$, $a_{n}^{m} \equiv 1, D_{0} y_{n}=y_{n}, D_{i} y_{n}=a_{n}^{i} \Delta D_{i-1} y_{n}, i=1,2, \ldots, m, \sigma(n) \rightarrow \infty$ as $n \rightarrow \infty$, and $f: \mathbb{R} \rightarrow \mathbb{R}$ is continuous with $u f(u)>0$ for $u \neq 0$. They give sufficient conditions to ensure that all bounded nonoscillatory solutions tend to zero as $n \rightarrow \infty$ without assuming that $\sum_{n=0}^{\infty} 1 / a_{n}^{i}=\infty, i=1,2, \ldots, m-1,\left\{q_{n}\right\}$ is positive, or $e_{n} \equiv 0$ as is often required. If $\left\{q_{n}\right\}$ is positive, they prove another such result for all nonoscillatory solutions.
\end{abstract}

2000 Mathematics Subject Classification. 39A10.

1. Introduction. Consider the $m$ th order nonlinear difference equation

$$
D_{m} y_{n}+q_{n} f\left(y_{\sigma(n)}\right)=e_{i},
$$

where $m \geq 1, n \in \mathbb{N}=\{0,1,2, \ldots\},\left\{a_{n}\right\},\left\{e_{n}\right\}$, and $\left\{a_{n}^{1}\right\},\left\{a_{n}^{2}\right\}, \ldots,\left\{a_{n}^{m-1}\right\}$ are real sequences, $a_{n}^{i}>0$ for $i=1,2, \ldots, m-1$ and all $n \in \mathbb{N}, a_{n}^{m} \equiv 1, D_{0} y_{n}=y_{n}, D_{i} y_{n}=$ $a_{n}^{i} \Delta D_{i-1} y_{n}$ for $i=1,2, \ldots, m,\{\sigma(n)\}$ is a sequence of positive integers with $\sigma(n) \rightarrow$ $\infty$ as $n \rightarrow \infty$, and $f: \mathbb{R} \rightarrow \mathbb{R}$ is continuous with $u f(u)>0$ for $u \neq 0$. Throughout, we will assume that

$$
\rho_{i}(n)=\sum_{s=n+1}^{\infty} \frac{\rho_{i-1}(s)}{a_{s}^{i}}, \quad i=1,2, \ldots, m-1, \rho_{0}(n) \equiv 1,
$$

satisfies

$$
\lim _{n \rightarrow \infty} \rho_{i}(n)=0 \text { for } i=1,2, \ldots, m-1 .
$$

Note that condition (1.3) is satisfied if

$$
\sum_{n=N}^{\infty} \frac{1}{a_{n}^{i}}<\infty \text { for each } i=1,2, \ldots, m-1 .
$$

By a solution of (1.1) we mean a nontrivial real sequence $\left\{y_{n}\right\}$ defined for $n \geq$ $N_{0}-\min _{n \in \mathbb{N}} \sigma(n), N_{0} \in \mathbb{N}$, and satisfying (1.1) for $n \geq N_{0}$. Such a solution is said to be oscillatory if for every $N \in \mathbb{N}$ there exist $n_{1}, n_{2} \in \mathbb{N}$ with $n_{2}>n_{1}>N$ and $y_{n_{1}} y_{n_{2}} \leq 0$, and it is said to be nonoscillatory otherwise.

An important problem in the study of oscillation theory of difference equations is to determine sufficient conditions for all nonoscillatory solutions or all bounded nonoscillatory solutions to converge to zero as $n \rightarrow \infty$. This problem has received a 
good deal of attention in the literature, and for recent results of this type, we refer the reader to the monographs of Agarwal [1], Agarwal and Wong [2] as well as the papers of Cheng et al. [3], Graef et al. [4], Graef and Spikes [5, 6], Szmanda [7], Thandapani and Lalli [8], Thandapani and Pandian [9], and Zhang [10]. Most of these results, however, are obtained under the assumptions that $\sum_{n=N}^{\infty} 1 / a_{n}^{i}=\infty, i=1,2, \ldots, m-1$, and/or $e_{n} \equiv 0$. It is these last two restrictions that provide the motivation for our work here. That is, we do not require that either of these conditions hold in our results below.

Our results are of two types. First, if the sequence $\left\{q_{n}\right\}$ is allowed to oscillate, we provide sufficient conditions for all bounded nonoscillatory solutions of (1.1) to converge to zero as $n \rightarrow \infty$. Second, in the case where $\left\{q_{n}\right\}$ is a nonnegative sequence, we give sufficient conditions for all nonoscillatory solutions of (1.1) to approach zero as $n \rightarrow \infty$. Examples to illustrate our results are also included.

2. Asymptotic decay of nonoscillatory solutions. We begin with a lemma that will be used in the proofs of our main results.

LEMMA 2.1. Consider the difference equation

$$
\Delta u_{n}-\frac{\Delta \rho(n)}{\rho(n)} u_{n}+\frac{\Delta \rho(n)}{\rho(n)} \phi_{n}=0,
$$

where $\left\{\phi_{n}\right\}$ and $\{\rho(n)\}$ are real sequences defined for $n \geq N$, for some $N \in \mathbb{N}$,

$$
\rho(n)>0, \quad \Delta \rho(n)<0, \quad \lim _{n \rightarrow \infty} \rho(n)=0 .
$$

Let $\left\{u_{n}\right\}$ be the solution of (2.1) defined for $n \geq N$ and satisfying $u_{N}=0$. Then

$$
\lim _{n \rightarrow \infty} \phi_{n}=\infty(-\infty) \text { implies } \lim _{n \rightarrow \infty} u_{n}=\infty(-\infty) .
$$

Proof. The solution $\left\{u_{n}\right\}$ of (2.1) is given by

$$
u_{n}=-\rho(n) \sum_{s=N}^{n-1} \frac{\Delta \rho(s)}{\rho(s) \rho(s+1)} \phi_{s}, \quad n \geq N .
$$

If $\lim _{n \rightarrow \infty} \phi_{n}=\infty(-\infty)$, then clearly

$$
\lim _{n \rightarrow \infty} \sum_{s=N}^{n-1} \frac{\Delta \rho(s)}{\rho(s) \rho(s+1)} \phi_{s}=-\infty(\infty) .
$$

Hence, by Stolz's theorem [1],

$$
\lim _{n \rightarrow \infty} u_{n}=\lim _{n \rightarrow \infty}\left|\frac{\Delta\left(-\sum_{s=N}^{n-1}(\Delta \rho(s) / \rho(s) \rho(s+1)) \phi_{s}\right)}{\Delta(1 / \rho(n))}\right|=\lim _{n \rightarrow \infty} \phi_{n}=\infty(-\infty),
$$

and this completes the proof of the lemma.

In our results that follow, we will make use of the notation $q_{n}^{+}=\max \left\{q_{n}, 0\right\}$ and $q_{n}^{-}=\max \left\{-q_{n}, 0\right\}$. 
THEOREM 2.2. Assume that

$$
\begin{gathered}
\sum_{n=N}^{\infty} \rho_{m-1}(n) q_{n}^{+}=\infty, \\
\sum_{n=N}^{\infty} \rho_{m-1}(n) q_{n}^{-}<\infty, \\
\sum_{n=N}^{\infty} \rho_{m-1}(n)\left|e_{n}\right|<\infty .
\end{gathered}
$$

Then all bounded nonoscillatory solutions of (1.1) tend to zero as $n \rightarrow \infty$.

Proof. Let $\left\{y_{n}\right\}$ be a bounded nonoscillatory solution of (1.1). Without loss of generality, we may assume that $y_{n}>0$ and $y_{\sigma(n)}>0$ for $n \geq N_{1}$ for some $N_{1} \in \mathbb{N}$. Define

$$
G_{0}(n)=y_{n}, \quad G_{i}(n)=a_{n}^{i} \Delta G_{i-1}(n), \quad i=1,2, \ldots, m-1,
$$

and observe that

$$
G_{i}(n)=D_{i} y_{n} \quad \text { for } i=1,2, \ldots, m-1, \quad \Delta G_{m-1}(n)=D_{m} y_{n}
$$

Next, we define the family of sequences

$$
u_{k}(n)=\sum_{s=N_{1}+1}^{n} \rho_{m-k-1}(s) \Delta G_{m-k-1}(s), \quad k=0,1, \ldots, m-1,
$$

for $n \geq N_{1}+1$.

A summation by parts yields

$$
\begin{aligned}
u_{k-1}(n)= & \sum_{s=N_{1}+1}^{n} \rho_{m-k}(s) \Delta G_{m-k}(s)=\rho_{m-k}(n+1) G_{m-k}(n+1) \\
& -\rho_{m-k}\left(N_{1}+1\right) G_{m-k}\left(N_{1}+1\right)+\sum_{s=N_{1}+1}^{n} \frac{\rho_{m-k-1}(s)}{a_{s}^{m-k}} G_{m-k}(s) \\
= & -\frac{\rho_{m-k}(n+1)}{\Delta \rho_{m-k}(n)} \Delta u_{k}(n)+\Delta u_{k}(n)+u_{k}(n)-2 \rho_{m-k}\left(N_{1}+1\right) G_{m-k}\left(N_{1}+1\right) \\
= & -\frac{\rho_{m-k}(n)}{\Delta \rho_{m-k}(n)} \Delta u_{k}(n)+u_{k}(n)-2 \rho_{m-k}\left(N_{1}+1\right) G_{m-k}\left(N_{1}+1\right) .
\end{aligned}
$$

This shows that each sequence $\left\{u_{k}(n)\right\}, k=0,1, \ldots, m-1$, satisfies the difference equation

$$
\frac{\rho_{m-k}(n)}{\Delta \rho_{m-k}(n)} \Delta u_{k}(n)-u_{k}(n)+\phi_{k}(n)=0,
$$

which can be written in the form

$$
\Delta u_{k}(n)-\frac{\Delta \rho_{m-k}(n)}{\rho_{m-k}(n)} u_{k}(n)+\frac{\Delta \rho_{m-k}(n)}{\rho_{m-k}(n)} \phi_{k}(n)=0,
$$


where $\phi_{k}(n)=u_{k-1}(n)+2 \rho_{m-k}\left(N_{1}+1\right) G_{m-k}\left(N_{1}+1\right)$. Since $u_{k}\left(N_{1}\right)=0$ by (2.11) and since $\rho_{m-k}(n)>0, \Delta \rho_{m-k}(n)<0$, and $\lim _{n \rightarrow \infty} \rho_{m-k}(n)=0$ by (1.3), we can apply Lemma 2.1 to (2.14) to conclude that $\lim _{n \rightarrow \infty} u_{k-1}(n)=\infty$ (or $-\infty$ ) which in turn implies that $\lim _{n \rightarrow \infty} u_{k}(n)=\infty$ (or $\left.-\infty\right)$.

Multiplying (1.1) by $\rho_{m-1}(n)$ and summing from $N_{1}+1$ to $n$, we have

$$
\begin{array}{r}
\sum_{s=N_{1}+1}^{n} \rho_{m-1}(s) \Delta G_{m-1}(s)+\sum_{s=N_{1}+1}^{n} \rho_{m-1}(s) q_{s}^{+} f\left(y_{\sigma(s)}\right) \\
=\sum_{s=N_{1}+1}^{n} \rho_{m-1}(s) e_{s}+\sum_{s=N_{1}+1}^{n} \rho_{m-1}(s) q_{s}^{-} f\left(y_{\sigma(s)}\right) .
\end{array}
$$

We consider the following two cases:

$$
\sum_{n=N_{1}+1}^{\infty} \rho_{m-1}(n) q_{n}^{+} f\left(y_{\sigma(n)}\right)=\infty
$$

or

$$
\sum_{n=N_{1}+1}^{\infty} \rho_{m-1}(n) q_{n}^{+} f\left(y_{\sigma(n)}\right)<\infty .
$$

Suppose (2.16) holds. In view of (2.8) and the boundedness of $\left\{y_{n}\right\}$, the right-hand side of (2.15) tends to a finite limit as $n \rightarrow \infty$. From (2.15), we see that $\lim _{n \rightarrow \infty} u_{0}(n)=-\infty$. Hence, applying Lemma 2.1 to (2.14) with $k=1$, we have $\lim _{n \rightarrow \infty} u_{1}(n)=-\infty$. Again applying Lemma 2.1 to (2.14), this time with $k=2$, we see that $\lim _{n \rightarrow \infty} u_{2}(n)=-\infty$. Repeating this procedure, we can conclude that $\lim _{n \rightarrow \infty} u_{m-1}(n)=-\infty$, which implies that $\lim _{n \rightarrow \infty} y_{n}=-\infty$. This, however, contradicts the assumption that $\left\{y_{n}\right\}$ is positive, and thus (2.16) cannot hold.

Next, letting $n \rightarrow \infty$ in (2.15) and using (2.17), we see that $\lim _{n \rightarrow \infty} u_{0}(n)$ is finite. From (2.13), with $k=1$, we have

$$
\frac{\rho_{m-1}(n)}{\Delta \rho_{m-1}(n)} \Delta u_{1}(n)=u_{1}(n)-\phi_{1}(n)
$$

or

$$
u_{1}(n)=\frac{\rho_{m-1}(n)}{\rho_{m-1}\left(N_{1}\right)}\left(u_{1}\left(N_{1}\right)-\rho_{m-1}\left(N_{1}\right) \sum_{s=N_{1}}^{n-1} \frac{\Delta \rho_{m-1}(s)}{\rho_{m-1}(s) \rho_{m-1}(s+1)} \phi_{1}(s)\right) .
$$

Taking the limit as $n \rightarrow \infty$ and using (1.3), we obtain

$$
\lim _{n \rightarrow \infty} u_{1}(n)=-\lim _{n \rightarrow \infty} \rho_{m-1}(n) \sum_{s=N_{1}}^{n-1} \frac{\Delta \rho_{m-1}(s)}{\rho_{m-1}(s) \rho_{m-1}(s+1)} \phi_{1}(s) .
$$

This limit must be finite since $\lim _{n \rightarrow \infty} u_{1}(n)=-\infty$ implies $\lim _{n \rightarrow \infty} y_{n}=-\infty$, which contradicts the positivity of $\left\{y_{n}\right\}$, and $\lim _{n \rightarrow \infty} u_{1}(n)=\infty \operatorname{implies} \lim _{n \rightarrow \infty} y_{n}=\infty$, which contradicts the boundedness of $\left\{y_{n}\right\}$. Continuing in this way, $\lim _{n \rightarrow \infty} u_{m-1}(n)$ is finite. Therefore, $\lim _{n \rightarrow \infty} y_{n}$ exists as a finite number. On the other hand, in view of (2.7) and (2.17), it is easy to verify that

$$
\liminf _{n \rightarrow \infty} y_{\sigma(n)}=\liminf _{n \rightarrow \infty} y_{n}=0 .
$$

Thus, it follows that $\lim _{n \rightarrow \infty} y_{n}=0$, and this completes the proof of the theorem. 
EXAMPLE 2.3. Consider the difference equation

$$
\Delta\left(n \Delta\left(n \Delta\left(n(n+1) \Delta y_{n}\right)\right)\right)+y_{\gamma n}=\frac{1}{\gamma n}, \quad n \geq 1,
$$

where $\gamma$ is a positive integer. We have $\rho_{1}(n)=\rho_{2}(n)=\rho_{3}(n)=1 /(n+1)$ and we see that all conditions of Theorem 2.2 are satisfied. Hence, all bounded nonoscillatory solutions of (2.22) tend to zero as $n \rightarrow \infty$. In fact, $\left\{y_{n}\right\}=\{1 / n\}$ is a solution of (2.22) having this property.

In the following theorem, we show that the conclusion of Theorem 2.2 still holds if the roles of the sequences $\left\{q_{n}^{+}\right\}$and $\left\{q_{n}^{-}\right\}$are interchanged.

THEOREM 2.4. All bounded nonoscillatory solutions of (1.1) tend to zero as $n \rightarrow \infty$ if the following conditions are satisfied:

$$
\begin{aligned}
& \sum_{n=N}^{\infty} \rho_{m-1}(n) q_{n}^{+}<\infty \\
& \sum_{n=N}^{\infty} \rho_{m-1}(n) q_{n}^{-}=\infty \\
& \sum_{n=N}^{\infty} \rho_{m-1}(n)\left|e_{n}\right|<\infty
\end{aligned}
$$

Proof. Let $\left\{y_{n}\right\}$ be a bounded nonoscillatory solution of (1.1), say, $y_{n}>0$ and $y_{\sigma(n)}>0$ for $n \geq N_{1} \geq N_{0}$. Define $G_{i}(n)$ and $u_{k}(n)$ as in (2.9) and (2.11). Assume that

$$
\sum_{n=N_{1}+1}^{\infty} \rho_{m-1}(n) q_{n}^{-} f\left(y_{\sigma(n)}\right)=\infty .
$$

Letting $n \rightarrow \infty$ in (2.15) and using (2.23), (2.25), and the boundedness of $\left\{y_{n}\right\}$, we obtain $\lim _{n \rightarrow \infty} u_{0}(n)=\infty$. Applying Lemma 2.1 to (2.14) with $k=1$, we see that $\lim _{n \rightarrow \infty} u_{1}(n)=\infty$. Repeated applications of this argument yield $\lim _{n \rightarrow \infty} u_{m-1}(n)=\infty$, which implies that $\lim _{n \rightarrow \infty} y_{n}=\infty$. This contradicts the boundedness of $\left\{y_{n}\right\}$, and so we must have

$$
\sum_{n=N_{1}+1}^{\infty} \rho_{m-1}(n) q_{n}^{-} f\left(y_{\sigma(n)}\right)<\infty .
$$

The remainder of the proof is similar to the proof of Theorem 2.2 and will be omitted.

EXAMPLE 2.5. Consider the equation

$$
\Delta^{4}\left(2^{n+1} \Delta y_{n}\right)-2^{n} y_{n-2}^{3}=-\frac{1}{4^{n-3}}, \quad n \geq 0 .
$$

It is easy to verify that the hypotheses of Theorem 2.4 are satisfied with $\rho_{1}(n)=$ $\rho_{2}(n)=\rho_{3}(n)=1 / 2^{n+1}$. It follows that all bounded nonoscillatory solutions of (2.28) approach zero as $n \rightarrow \infty$. One such solution is $\left\{y_{n}\right\}=\left\{1 / 2^{n}\right\}$.

As an example where $\left\{q_{n}\right\}$ is oscillatory, we have the following example. 
EXAMPLE 2.6. Consider the equation

$$
\begin{aligned}
& \Delta^{3}\left(2^{n+1} \Delta y_{n}\right)+\left\{2^{n-1}\left[1+(-1)^{n}\right]-\frac{\left[1+(-1)^{n+1}\right]}{2 n^{2}}\right\} y_{n} \\
&=\frac{\left[1+(-1)^{n}\right]}{2}-\frac{\left[1+(-1)^{n+1}\right]}{2^{n+1} n^{2}}, \quad n \geq 1 .
\end{aligned}
$$

Observe that $q_{n}^{+}=2^{n}, q_{n}^{-}=-1 / n^{2}$, and $\rho_{1}(n)=\rho_{2}(n)=\rho_{3}(n)=1 / 2^{n+1}$. All the hypotheses of Theorem 2.2 are satisfied so all bounded nonoscillatory solutions of (2.29) approach zero as $n \rightarrow \infty$. Here, $\left\{y_{n}\right\}=\left\{1 / 2^{n}\right\}$ is such a solution. Clearly, a simple modification of this equation will yield an example of Theorem 2.4.

In our final result, we examine (1.1) in the case where $\left\{q_{n}\right\}$ is positive and establish conditions under which all nonoscillatory solutions are bounded and tend to zero as $n \rightarrow \infty$.

THEOREM 2.7. Assume that condition (1.4) holds, $\left\{q_{n}\right\}$ is positive, $\liminf _{u \rightarrow \infty} f(u)>0$, and $\lim \sup _{u \rightarrow-\infty} f(u)<0$. If

$$
\begin{gathered}
\sum_{n=N}^{\infty} \rho_{m-1}(n) q_{n}=\infty, \\
\sum_{n=N}^{\infty}\left|e_{n}\right|<\infty,
\end{gathered}
$$

then all nonoscillatory solutions of (1.1) tend to zero as $n \rightarrow \infty$.

Proof. Let $\left\{y_{n}\right\}$ be a nonoscillatory solution of (1.1), say, $y_{n}>0$ and $y_{\sigma(n)}>0$ for $n \geq N_{1} \geq N_{0}$. Define $G_{i}(n)$ and $u_{k}(n)$ as in (2.9) and (2.11). We will first show that $\left\{y_{n}\right\}$ is bounded above. From (1.1), we obtain

$$
G_{m-1}(n)-G_{m-1}\left(N_{1}\right)+\sum_{s=N_{1}}^{n-1} q_{s} f\left(y_{\sigma(s)}\right)=\sum_{s=N_{1}}^{n-1} e_{s} .
$$

Since the first sum in (2.32) is positive, and by (2.31), the second sum is bounded, there exists a constant $K_{m-1}$ such that

$$
G_{m-1}(n)=a_{n}^{m-1} \Delta G_{m-2}(n) \leq K_{m-1} \text { for } n \geq N_{1} .
$$

Dividing the last inequality by $a_{n}^{m-1}$ and summing from $N_{1}$ to $n-1$, we obtain

$$
G_{m-2}(n)-G_{m-2}\left(N_{1}\right) \leq K_{m-1} \sum_{s=N_{1}}^{n-1} \frac{1}{a_{s}^{m-1}} \text { for } n \geq N_{1},
$$

which, in view of (1.4), implies there exists a constant $K_{m-2}$ such that

$$
G_{m-2}(n)=a_{n}^{m-2} \Delta G_{m-3}(n) \leq K_{m-2} \text { for } n \geq N_{1} .
$$

Repeatedly applying the above argument, we obtain constants $K_{m-3}, \ldots, K_{1}, K_{0}$ such that

$$
G_{m-3}(n) \leq K_{m-3}, \ldots, G_{1}(n) \leq K_{1}, G_{0}(n) \leq K_{0} \text { for } n \geq N_{1} .
$$

It follows that $\left\{y_{n}\right\}$ is bounded from above. 
Now, we argue as in the proof of Theorem 2.2 using

$$
\sum_{s=N_{1}+1}^{n} \rho_{m-1}(s) \Delta G_{m-1}(s)+\sum_{s=N_{1}+1}^{n} \rho_{m-1}(s) q_{s} f\left(y_{\sigma(s)}\right)=\sum_{s=N_{1}+1}^{n} \rho_{m-1}(s) e_{s}
$$

in place of (2.15). Noting that (2.31) implies the right-hand side of (2.37) tends to a finite limit as $n \rightarrow \infty$, we claim that

$$
\sum_{s=N_{1}+1}^{n} \rho_{m-1}(s) q_{s} f\left(y_{\sigma(s)}\right)<\infty .
$$

If this was not the case, we could use Lemma 2.1 to obtain $\lim _{n \rightarrow \infty} u_{k}(n)=-\infty$ for $k=0,1, \ldots, m-1$, and contradict the boundedness of $\left\{y_{n}\right\}$. Next, using (2.37) and (2.38) we can show that $\lim _{n \rightarrow \infty} u_{k}(n)$ is finite for each $k=0,1, \ldots, m-1$. Thus, $\lim _{n \rightarrow \infty} y_{n}$ exists and is finite. On the other hand, from (2.30) and (2.38), we see that $\liminf _{n \rightarrow \infty} y_{n}=0$. Hence, $\left\{y_{n}\right\}$ tends to zero as $n \rightarrow \infty$, and this completes the proof of the theorem.

We conclude this paper with some examples of Theorem 2.7.

EXAMPLE 2.8. Consider the equation

$$
\Delta\left(2^{n} \Delta\left(2^{n} \Delta\left(2^{n} \Delta y_{n}\right)\right)\right)+8^{n} y_{n+k}^{3}=\frac{1}{8^{k}}, \quad n \geq 0,
$$

where $k$ is a positive integer. In this case, $\rho_{1}(n)=1 / 2^{n}, \rho_{2}(n)=(1 / 3)\left(1 / 4^{n}\right)$, and $\rho_{3}(n)=(1 / 21)\left(1 / 8^{n}\right)$. Since all conditions of Theorem 2.7 are satisfied, every nonoscillatory solution of (2.39) tends to zero as $n \rightarrow \infty$, and $\left\{y_{n}\right\}=\left\{1 / 2^{n}\right\}$ is such a solution.

EXAMPLE 2.9. Consider the equation

$$
\Delta\left(n(n+1) \Delta\left((n+2)(n+3) \Delta\left(n(n+1) \Delta y_{n}\right)\right)\right)+n^{4} y_{k n}^{3}=\frac{n}{k^{3}(k n+1)^{3}}, \quad n \geq 1,
$$

where $k$ is a positive integer. All the hypotheses of Theorem 2.7 are satisfied with $\rho_{1}(n)=1 /(n+1), \rho_{2}(n)=1 / 2(n+1)(n+2)$, and $\rho_{3}(n)=1 / 6(n+1)(n+2)(n+3)$, so every nonoscillatory solution of (2.40) tends to zero as $n \rightarrow \infty$. Here, $\left\{y_{n}\right\}=\{1 / n(n+1)\}$ is a solution of (2.40).

\section{REFERENCES}

[1] R. P. Agarwal, Difference Equations and Inequalities. Theory, Methods, and Applications, Monographs and Textbooks in Pure and Applied Mathematics, vol. 155, Marcel Dekker, New York, 1992. MR 92m:39002. Zbl 925.39001.

[2] R. P. Agarwal and P. J. Y. Wong, Advanced Topics in Difference Equations, Mathematics and its Applications, vol. 404, Kluwer, Dordrecht, 1997. MR 98i:39001. Zbl 878.39001.

[3] S. S. Cheng, H. J. Li, and W. T. Patula, Bounded and zero convergent solutions of second-order difference equations, J. Math. Anal. Appl. 141 (1989), no. 2, 463-483. MR 90g:39001. Zbl 698.39002.

[4] J. R. Graef, J. Jaroš, A. Miciano, P. W. Spikes, and E. Thandapani, Oscillation and nonoscillation results for general nonlinear difference equations, Proceedings of Dynamic Systems and Applications (Atlanta, GA, 1995) (G. S. Ladde et al., ed.), vol. 2, Dynamic, Georgia, 1996, pp. 199-206. MR 98a:39003. Zbl 861.39010. 
[5] J. R. Graef and P. W. Spikes, Asymptotic decay of oscillatory solutions of forced nonlinear difference equations, Dynam. Systems Appl. 3 (1994), no. 1, 95-102. MR 95a:39007. Zbl 790.39002.

[6] __ Boundedness and asymptotic behavior of solutions of a forced difference equation, Int. J. Math. Math. Sci. 17 (1994), no. 2, 397-400. MR 95a:39008. Zbl 810.39002.

[7] B. Szmanda, Nonoscillation, oscillation and growth of solutions of nonlinear difference equations of second order, J. Math. Anal. Appl. 109 (1985), no. 1, 22-30. MR 87e:39007. Zbl 589.39003.

[8] E. Thandapani and B. S. Lalli, Asymptotic behavior and oscillation of difference equations of Volterra type, Appl. Math. Lett. 7 (1994), no. 1, 89-93. MR 96i:39010. Zbl 792.39004 .

[9] E. Thandapani and S. Pandian, Oscillatory and asymptotic behavior of solutions of higher order nonlinear difference equations, Bull. Calcutta Math. Soc. 87 (1995), no. 3, 277-282. MR 97f:39010. Zbl 855.39012.

[10] B. G. Zhang, Oscillation and asymptotic behavior of second order difference equations, J. Math. Anal. Appl. 173 (1993), no. 1, 58-68. MR 94a:39010. Zbl 780.39006.

E. Thandapani AND S. Lourdu Marian: Department of Mathematics, Periyar UniverSITY, SALEM 636011, TAMIL NADU, INDIA

John R. Graef: Department of Mathematics, University of Tennessee at Chattanooga, ChatTanooga, TN 37403, USA

E-mail address: john-graef@utc.edu 


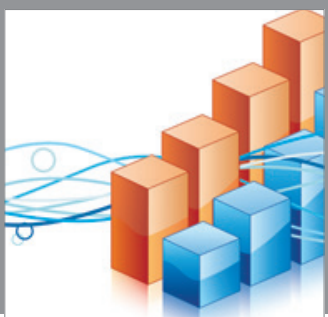

Advances in

Operations Research

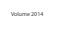

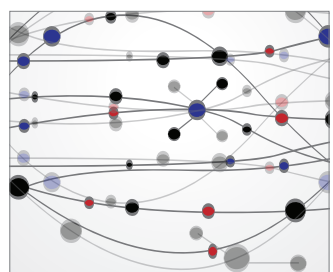

\section{The Scientific} World Journal
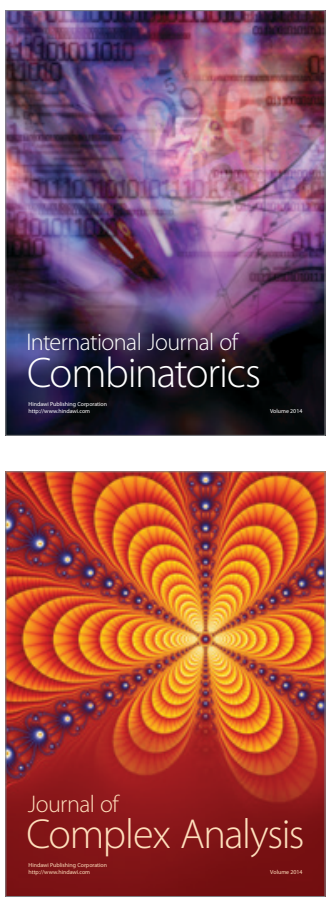

International Journal of

Mathematics and

Mathematical

Sciences
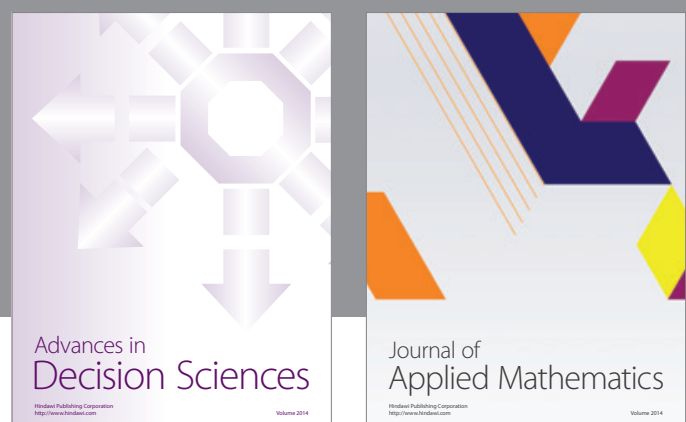

Journal of

Applied Mathematics
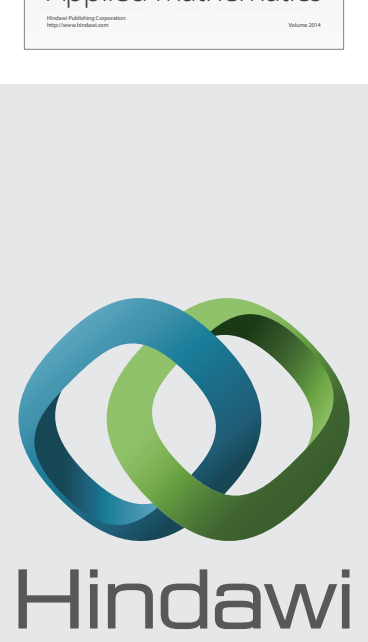

Submit your manuscripts at http://www.hindawi.com
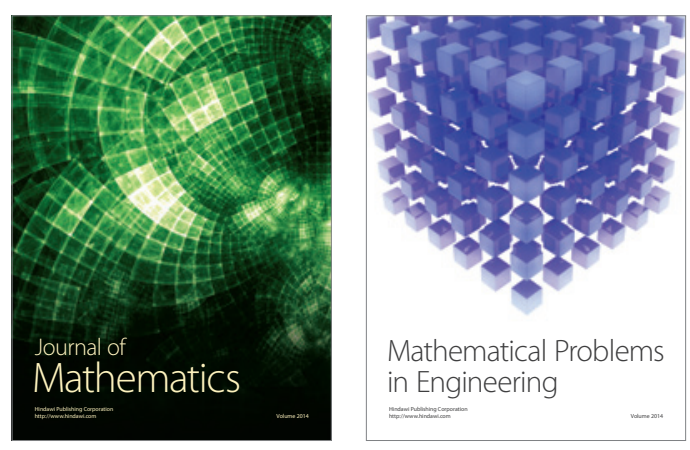

Mathematical Problems in Engineering
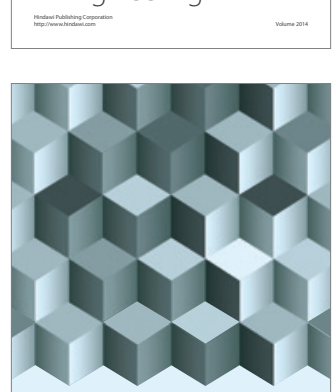

Journal of

Function Spaces
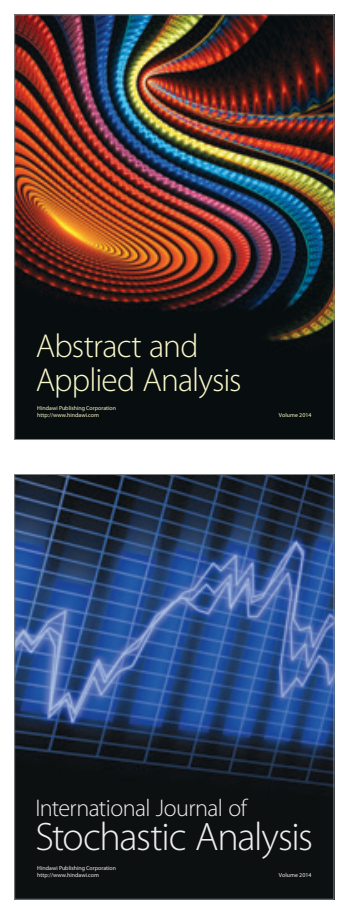

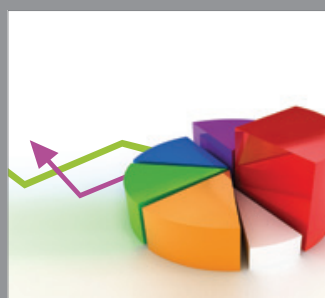

ournal of

Probability and Statistics

Promensencen
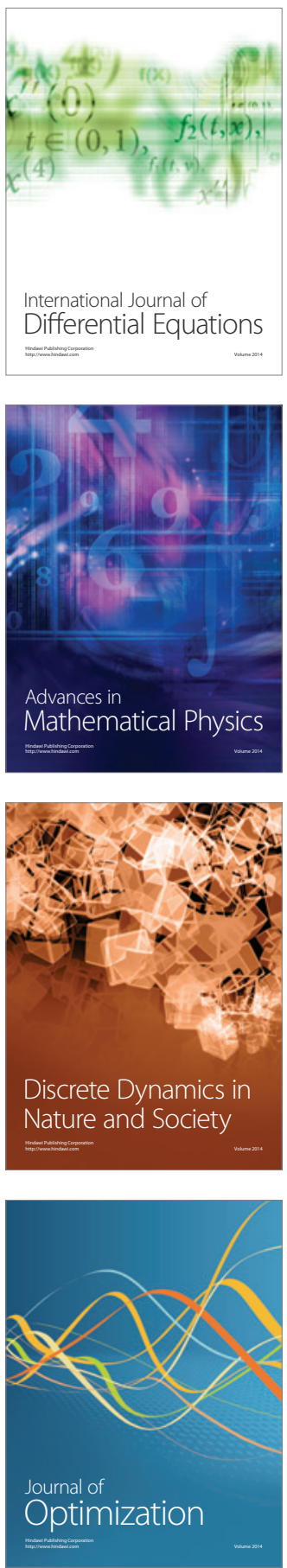